\title{
Dynamical Law for Slow Crack Growth in Polycarbonate Films
}

\author{
Pierre-Philippe Cortet, Loïc Vanel, ${ }^{*}$ and Sergio Ciliberto \\ Université de Lyon, Laboratoire de physique, Ecole Normale Supérieure de Lyon, CNRS, France
}

(Received 22 June 2007; published 14 November 2007)

\begin{abstract}
We study experimentally the slow growth of a single crack in polycarbonate films submitted to uniaxial and constant imposed stress. For this viscoplastic material, we uncover a dynamical law that describes the dependence of the instantaneous crack velocity with experimental parameters. The law involves a Dugdale-Barenblatt static description of crack tip plastic zones associated to an Eyring's law and an empirical dependence with the crack length that may come from a residual elastic field.
\end{abstract}

DOI: 10.1103/PhysRevLett.99.205502

PACS numbers: 62.20.Mk, 46.35.+z, 62.20.Fe

Stressed solids commonly break apart once a critical stress threshold is reached. However, many experiments [1-5] show that a given solid submitted to a subcritical stress breaks after a certain amount of time. Therefore, understanding the mechanisms of subcritical rupture of solids has become an important goal of fracture physics in order to improve the resistance of structures to delayed failure that may have catastrophic consequences. According to reported experimental works [1,2], the dependence of the rupture time with applied stress $\sigma$ can be described in many kinds of materials (polymers, metal alloys, semiconductors, rocks...) by an Arrhenius law with an energy barrier decreasing linearly with $\sigma$. This proposed universality is disturbing since these materials have microstructures and rheological properties very different from one another, and the rupture dynamics is certainly expected to be dependent on those properties. To lift this paradox, one must go beyond characterization of global properties such as rupture time and instead study experimentally the full time-resolved rupture dynamics, from the stress application to the final breakdown of the sample. A convenient system to start with is a two-dimensional solid with a single macroscopic initial crack submitted to a uniaxial constant load.

In this context, recent experimental studies [5] have shown that subcritical crack growth in paper sheets can be successfully described by a thermally activated mechanism inspired from previous theoretical works in elastic brittle media [6,7]. Experimental study of slow crack growth in a viscoplastic material under stress is a very active topic [8]. General theoretical frameworks [9-11] have been proposed to predict the dependence of the crack growth velocity with experimental parameters using characteristic material time-response functions such as its compliance. However, these models involve complex integrodifferential equations which are hardly tractable in practical situations where viscoplastic effects are strong. Consequently, the experimental time evolution of the instantaneous crack growth dynamics cannot be captured easily by current models.

In order to provide more experimental insight in our understanding of viscoplastic effects during slow crack growth, we have performed an experimental study of the slow growth of a single crack in amorphous polymer films made of polycarbonate, a highly nonbrittle viscoplastic material. The experiments consist of the growth of a single linear crack in a polycarbonate film submitted to uniaxial and constant imposed force. The polycarbonate films used are Bayer Makrofol@ DE and have the properties of bulk material. Before each experiment, a crack of length $\ell_{i}$ (from 0.5 to $3 \mathrm{~cm}$ ) is initiated at the center of the polycarbonate sample (height $H=21 \mathrm{~cm}$ (same direction as the crack), length $L=24 \mathrm{~cm}$, thickness $e=125 \mu \mathrm{m}$ ). Then, a constant force $F$ is applied to the film perpendicularly to the crack direction, so that we get a mode 1 crack opening configuration. Using a camera, we follow the growth of the crack length $\ell$ under constant applied stress $\sigma=F / e H$ until the total rupture of the sample. The applied stress $\sigma$ is chosen such that crack growth is slow, i.e., smaller than a critical one $\sigma_{c}$, above which crack propagation occurs in a few seconds. More details about the experimental setup can be found in [12].

In each crack growth experiment, during the loading phase of the film, a macroscopic flame-shaped plastic zone appears at each tip of the crack [13] and grows with the applied stress (cf. Fig. 1 where is defined the plastic zone length from tip to tip $\ell_{\mathrm{pz}}$ ). In the late loading stage, the crack may also start to grow at a time that appears to be statistical. It is probably a consequence of the dispersion in the local toughness of the material or in the initial crack tip shape. Consequently, the real experimental initial condition, obtained when the constant stress $\sigma$ is reached, is not exactly $\ell=\ell_{i}$. Depending on the moment when the crack starts to grow during the loading phase, the true initial condition of the creep experiment will be a couple of

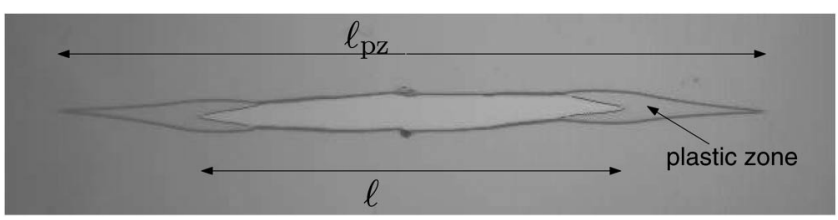

FIG. 1. Image of a crack in a polycarbonate film with its macroscopic plastic zone at each tip. 
values for the crack and plastic zone length (Fig. 1): $\left(\ell^{*}\right.$, $\left.\ell_{\mathrm{pz}}^{*}\right)$. Finally, during the imposed stress stage, the plastic zones and the crack are both growing until the final breakdown of the sample in a way that the crack never catches up to the plastic zone tip. Inside the plastic zone, the film is subjected to a thinning which brings its thickness down to $75 \pm 5 \mu \mathrm{m}$.

Typical growth curves of the fracture and plastic zone are shown in Fig. 2. Both curves show a quite similar smooth shape. This regular shape lets us think that the crack growth in polycarbonate films is a deterministic phenomenon. However, for identical experimental conditions, we notice a large dispersion of the rupture times and more generally of the crack growth dynamics. There is actually up to a factor five between the rupture time of the fastest and slowest experiments. We suggest that the explanation for this statistics in the crack growth dynamics does not come from the growth mechanism itself, but is a consequence of the dispersion in the effective initial conditions at the beginning of the constant stress phase of the experiment $\left(\ell^{*}, \ell_{\mathrm{pz}}^{*}\right)$. These initial conditions are clearly statistical and hardly controllable in our experiment. They are dependent on the moment when the crack starts growing during the loading stage of the sample and they determine all the rest of the experiment.

In Fig. 3, we show the evolution of the average rupture time $\left\langle T_{r}\right\rangle$ (averaged over at least ten experiments) as a function of the applied stress for a series of experiments with $\ell_{i}=1.5 \mathrm{~cm}$. We see a linear dependence of $\log \left\langle T_{r}\right\rangle$ with the applied stress that corresponds well to an exponential description of the rupture time as proposed by Zhurkov [1]. The linear fit of the data is of quite good quality and suggests that $\left\langle T_{r}\right\rangle=T_{0} e^{-\gamma \sigma}$. In Zhurkov's approach, the stress dependence of $\left\langle T_{r}\right\rangle$ is interpreted as an Eyring's law [14] with $\gamma=V / k_{B} T$, where $V$ is assumed to be a characteristic volume of the material. However, in our experiments, the parameter $V$ cannot be a constant since a different initial crack length $\ell_{i}$ gives a completely

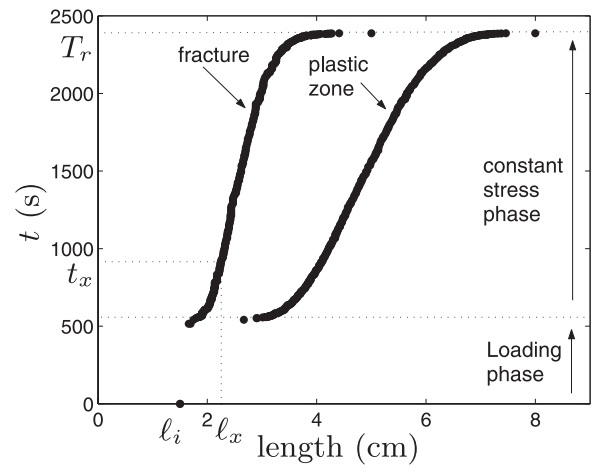

FIG. 2. Time as a function of both the crack and process zone lengths for an imposed stress experiment $\left(\ell_{i}=1.5 \mathrm{~cm}, F=\right.$ $900 \mathrm{~N})$. We indicate the position of the inflection point $\left(t_{x}, \ell_{x}\right)$ of the crack growth curve. different rupture time for the same applied stress. Thus, the external applied stress $\sigma$ cannot be the single control parameter of the rupture dynamics. Then, it is clear that Zhurkov's description needs to be improved to take into account the specific geometry of the problem. In particular, the stress $\sigma_{y}$ holding in the plastic zone close to the crack tips most probably participates in the dynamical processes leading to the crack growth.

The Dugdale-Barenblatt cohesive zone model $[15,16]$ is a good and simple means to estimate the stress $\sigma_{y}$. This quantity appears intuitively as a possible control parameter for the crack dynamics just like the stress intensity factor is for brittle materials. The model predicts

$$
\sigma_{y}=\frac{\pi}{2} \frac{\sigma}{\operatorname{arcos}\left(\frac{\ell}{\ell_{\mathrm{p} 2}}\right)} .
$$

This plastic stress $\sigma_{y}$ can be computed at each moment using Eq. (1) with the instantaneous values of $\sigma, \ell$ and $\ell_{\mathrm{pz}}$. To account for the global dynamics during an experiment, we compute the time-averaged growth velocity on the whole experiment $\bar{v}$ and compare it to the time-averaged plastic stress $\overline{\sigma_{y}}$ (see Fig. 4). Each point of this Figure represents the mean behavior over an experiment. The data are compatible with a linear law that predicts an exponential dependence of the average growth velocity with the mean stress in the plastic zone

$$
\bar{v}=v_{0} e^{a \overline{y_{y}}},
$$

with $a=6.3 \times 10^{-7} \mathrm{~m}^{2} \mathrm{~N}^{-1}$ and $v_{0}=7.8 \times 10^{-21} \mathrm{~m} \mathrm{~s}^{-1}$.

We now go beyond a simple analysis of the average growth dynamics by looking at the dependence of the crack velocity with the stress in the plastic zone at each time during the crack growth. We plot in Fig. 5(a) the instantaneous crack velocity $v=d \ell / d t$ as a function of the instantaneous value of the Dugdale-Barenblatt stress $\sigma_{y}$ for eight experiments performed with various experimental conditions. Here, the description of the instantaneous velocity by an exponential law fails, especially when the crack length becomes larger than $\ell_{x}$ at which the minimum

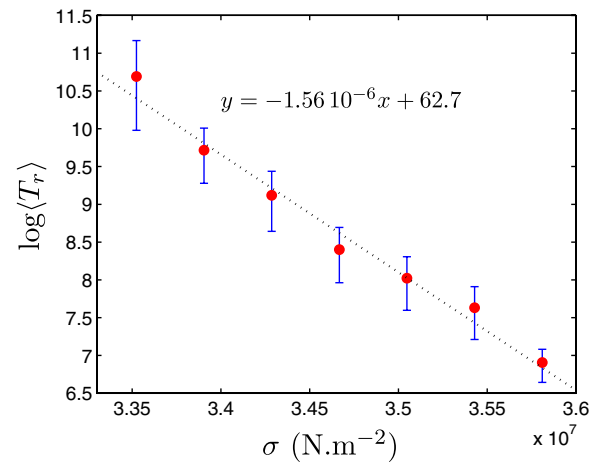

FIG. 3 (color online). Natural logarithm of the average rupture time as a function of the applied stress for a series of experiments performed for $\ell_{i}=1.5 \mathrm{~cm}$. 


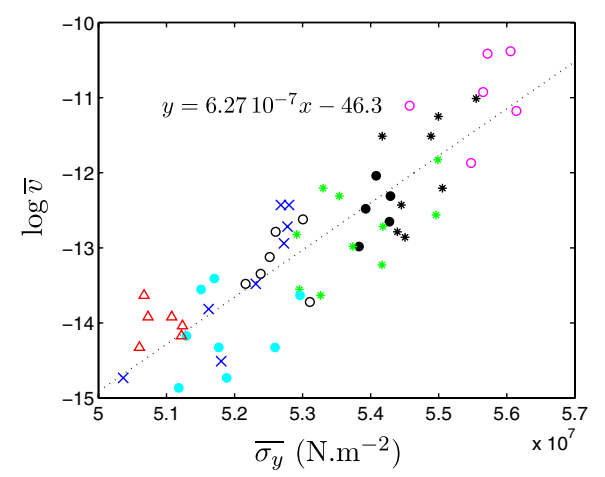

FIG. 4 (color online). Natural logarithm of the average crack growth velocity $\bar{v}$ as a function of the average plastic stress during the growth. Each point represents the average dynamical behavior during an experiment. Experimental conditions are various $\left(\ell_{i}=1.5,2,3 \mathrm{~cm}\right.$ and $\left.2.9<\sigma<3.8 \times 10^{7} \mathrm{~N} \mathrm{~m}^{-2}\right)$. Each experimental condition corresponds to different symbols.

crack velocity is reached $\left(\ell_{x}\right.$ is also the inflexion point of the growth curve in Fig. 2). In fact, the law given by Eq. (2) describes well the behavior only when $\ell \simeq \ell_{x}$. We discovered that introducing a correction to $\sigma_{y}$ linear with the crack length $\ell$ allows us to collapse the experimental data on a straight line [cf. Fig. 5(b)]. This correction can be written as
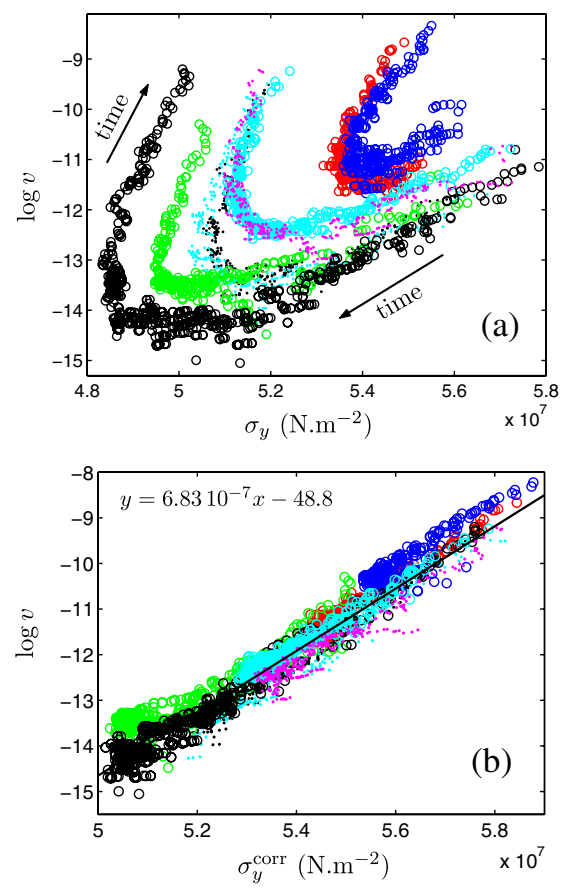

FIG. 5 (color online). Natural logarithm of the instantaneous crack growth velocity as a function of (a) the Dugdale-Barenblatt stress, (b) the corrected Dugdale-Barenblatt stress $\sigma_{y}^{\text {corr }}$ according to Eq. (3) for eight experiments performed with various experimental conditions $\left(\ell_{i}=1.5,2,3 \mathrm{~cm}\right.$ and $2.9<\sigma<3.8 \times$ $10^{7} \mathrm{~N} \mathrm{~m}^{-2}$ ). In Fig. (b), the black line is the result of a linear data fit.

$$
\sigma_{y}^{\mathrm{corr}}=\frac{\pi}{2} \frac{\sigma}{\operatorname{arcos}\left(\frac{\ell}{\ell_{\mathrm{pz}}}\right)}+\kappa\left(\ell-\ell_{x}\right) .
$$

For each experiment, we determine the value $\kappa=(3.4 \pm$ $0.6) \times 10^{8} \mathrm{~N} \mathrm{~m}^{-3}$. The dispersion of $\kappa$ values seems to be statistical as no systematic dependence with $\sigma$ or $\ell_{i}$ could be found. This rescaling means that the crack growth velocity seems to follow

$$
v=v_{0} e^{\alpha \sigma_{y}^{\text {corr }}}
$$

where $\alpha=6.810^{-7} \mathrm{~m}^{2} \mathrm{~N}^{-1}$. The collapse of the data for various experimental conditions means that $v_{0}$ can be considered as a constant.

In Eq. (3), the crack length at the inflexion point in the growth curve plays a particular role. It turns out that its value depends on the experimental conditions. This can be seen in Fig. 6(a) where the product $\kappa \ell_{x}$, with $\kappa=3.4 \times$ $10^{8} \mathrm{~N} \mathrm{~m}^{-3}$, is plotted as a function of the applied stress $\sigma$. Remarkably, the dependence of $\kappa \ell_{x}$ with $\sigma$ is well approximated by a linear fit: $\kappa \ell_{x}=\sigma_{x}-\sigma$, where $\sigma_{x}=$ $4.2 \times 10^{7} \mathrm{~N} \mathrm{~m}^{-2}$ is only an estimate of $\kappa \ell_{x}$ for $\sigma=0$.

A way to clarify the meaning of this relation is to look at the dependence of the critical stress $\sigma_{c}$ needed to break instantaneously a sample with a crack of initial length $\ell_{i}$. In brittle materials, we would expect this critical stress to decrease in $1 / \sqrt{\ell_{i}}$ since the rupture criterion is reached when the initial stress intensity factor equals the toughness
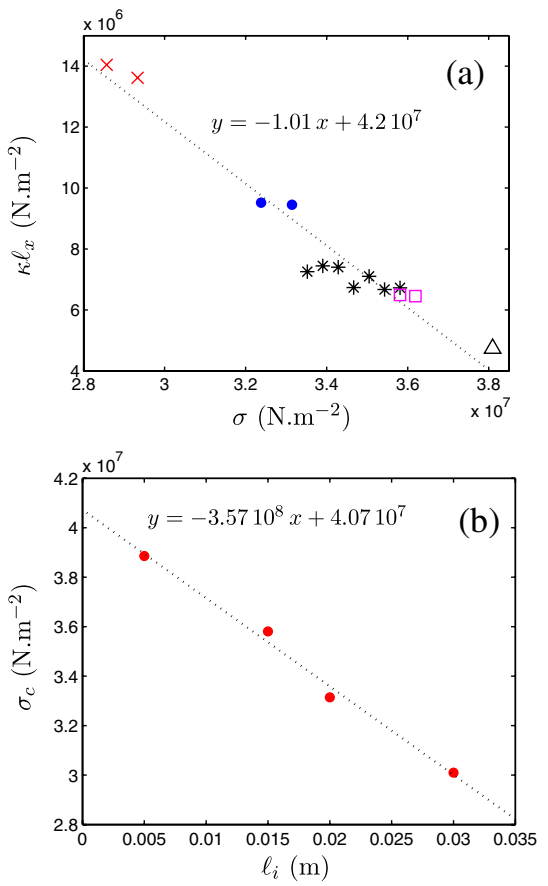

FIG. 6 (color online). (a) $\kappa \ell_{x}$ for various experimental conditions $\left(\ell_{i}=1.5,2,3 \mathrm{~cm}\right.$ and $\left.2.9<\sigma<3.8 \times 10^{7} \mathrm{~N} \mathrm{~m}^{-2}\right)$ as a function of the applied stress $\sigma$. (b) Critical rupture stress $\sigma_{c}$ as a function of the initial crack length $\ell_{i}$. The dotted lines are a linear fit of the data. 
of the material $K_{c}$ [5]: $\sigma_{c} \sqrt{\pi \ell_{i}} / 2=K_{c}$. For an amorphous viscoplastic material such as polycarbonate, we do not get the same functional dependence. Indeed, as we can see in Fig. 6(b), the relation between $\sigma_{c}$ and $\ell_{i}$ can be approximated by a linear equation: $\beta \ell_{i}=\sigma_{s}-\sigma_{c}$, where $\sigma_{s}=$ $4.07 \times 10^{7} \mathrm{~N} \mathrm{~m}^{-2}$ and $\beta=3.57 \times 10^{8} \mathrm{~N} \mathrm{~m}^{-3}$. We note that $\kappa \simeq \beta$ and $\sigma_{x} \simeq \sigma_{s}$ and will consider these quantities to be the same material constants. So, we find that the quantity $\Sigma\left(\sigma_{c}, \ell_{i}\right)=\sigma_{c}+\kappa \ell_{i}$ may play a role similar to the initial stress intensity factor in brittle materials. Furthermore, it allows us to interpret the value of the crack length at the inflexion point as defined by a characteristic value of the quantity $\Sigma\left(\sigma, \ell_{x}\right)=\sigma_{x} \simeq \sigma_{s}$ that corresponds to an intrinsic property of polycarbonate. Indeed, $\sigma_{s}$ is simply the value of the critical rupture stress $\sigma_{c}$ when there is no initial crack.

Finally, the effective stress $\sigma_{y}^{\text {corr }}$ may be rewritten as

$$
\sigma_{y}^{\text {corr }}=\frac{\pi}{2} \frac{\sigma}{\operatorname{arcos}\left(\frac{\ell}{\ell_{\mathrm{pz}}}\right)}+\kappa \ell+\sigma-\sigma_{s},
$$

where we clearly see that it is composed of, the DugdaleBarenblatt estimation of the crack tip plastic zone stress $\sigma_{y}$, a linear dependence with the crack length $\kappa \ell$ and the applied stress at the borders of the sample $\sigma$.

Furthermore, one can interpret the exponential dependence of the velocity as a function of $\sigma_{y}^{\text {corr }}$ [cf. Eq. (4)] in a rather simple and physical way. Indeed, polycarbonate creep is known to obey an Eyring's law relating its strain rate $\dot{\epsilon}$ to the applied stress $\sigma: \dot{\epsilon}=\dot{\epsilon}_{1} \exp \left(\sigma V / k_{B} T\right)$ [17]. Creep experiments performed at room temperature on our own polycarbonate samples give $V / k_{B} T=7.6 \times$ $10^{-7} \mathrm{~m}^{2} \mathrm{~N}^{-1}$ [18]. The fact this prefactor $V / k_{B} T$ is of the same magnitude than $\alpha$ in the exponential law for the crack velocity [cf. Eq. (4)] suggests that the Eyring's law plays an important role in the mechanisms of crack growth. In order to possibly identify $\alpha$ with $V / k_{B} T$, it remains to check the dependence of $\alpha$ on temperature. This will be discussed in more detail in [18].

Conclusion. - We have studied experimentally the slow growth of a single crack in polycarbonate films submitted to uniaxial and constant imposed stress. The main result of this analysis is that the instantaneous crack velocity appears to be ruled, during an experiment, by an exponential law [cf. Eq. (4)] with an effective stress $\sigma_{y}^{\text {corr }}$ given by Eq. (5).

In this equation, the viscous relaxation is taken into account by the experimentally measured evolution of the ratio $\ell / \ell_{p z}$ as the crack grows. Indeed, if this ratio was constant, the stress in the plastic zone would also be constant and the velocity would increase monotonously due to the linear term in crack length. In that case, the behavior would actually be qualitatively the same as the one for crack growth in brittle facture [5]. To predict fully the viscous dynamics of the crack, we need a second equation that will prescribe $\ell_{\mathrm{pz}}$ :

$$
\frac{d \ell_{\mathrm{pz}}}{d t}=f\left(\ell_{\mathrm{pz}}, \ell, \dot{\ell}, \sigma, \ldots\right)
$$

An original theoretical approach recently developed by Bouchbinder [19] based on the shear-transformation-zone theory proposed by Falk and Langer [20] is certainly useful for deriving an equation of the plastic zone velocity [cf. Eq. (6)]. Additionally, numerical simulations that can reproduce the complex viscoplastic behavior of polycarbonate may help in going further in the interpretation of our experimental results [21-23].

*Electronic address: Loic.Vanel@ens-lyon.fr

[1] S. N. Zhurkov, Int. J. Fracture Mech. 1, 311 (1965).

[2] F. Bueche, J. Appl. Phys. 26, 1133 (1955); 28, 784 (1957).

[3] S. S. Brenner, J. Appl. Phys. 33, 33 (1962).

[4] L. Pauchard and J. Meunier, Phys. Rev. Lett. 70, 3565 (1993).

[5] S. Santucci, L. Vanel, and S. Ciliberto, Phys. Rev. Lett. 93, 095505 (2004); S. Santucci et al., Europhys. Lett. 74, 595 (2006).

[6] C. Hsieh and R. Thomson, J. Appl. Phys. 44, 2051 (1973); M. Marder, Phys. Rev. E 54, 3442 (1996).

[7] S. Santucci et al., Europhys. Lett. 62, 320 (2003).

[8] N. Haddaoui, A. Chudnovsky, and A. Moet, Polymer 27, 1377 (1986); M. T. Takemory and R. P. Kambour, J. Mater. Sci. 16, 1108 (1981); S. Hashemi and J. G. Williams, Plast. Rubber Compos. 29, 294 (2000).

[9] R. A. Schapery, Int. J. Fracture 11, 141 (1975); 11, 369 (1975); 11, 549 (1975).

[10] A. A. Kaminskii, Sov. Appl. Mech. 15, 1078 (1979); Int. Appl. Mech. 40, 829 (2004).

[11] A. Chudnovsky and Y. Shulkin, Int. J. Fract. 97, 83 (1999).

[12] P.-P. Cortet, S. Santucci, L. Vanel, and S. Ciliberto, Europhys. Lett. 71, 242 (2005).

[13] A. M. Donald and E. J. Kramer, J. Mater. Sci. 16, 2967 (1981).

[14] H. Eyring, J. Chem. Phys. 4, 283 (1936).

[15] D. S. Dugdale, J. Mech. Phys. Solids 8, 100 (1960).

[16] G. I. Barenblatt, Advances in Applied Mechanics (Academic, New York, 1962), Vol. 7, p. 55.

[17] C. Bauwens-Crowet, J.C. Bauwens, and G. Homes, J. Mater. Sci. 7, 176 (1972).

[18] P.-P. Cortet, L. Vanel, and S. Ciliberto (to be published).

[19] E. Bouchbinder, J.S. Langer, and I. Procaccia, Phys. Rev. E 75, 036107 (2007); 75, 036108 (2007).

[20] M. L. Falk, J. S. Langer, and L. Pechenik, Phys. Rev. E 70, 011507 (2004).

[21] M.C. Boyce, E. L. Montagut, and A.S. Argon, Polym. Eng. Sci. 32, 1073 (1992).

[22] B. P. Gearing and L. Anand, Int. J. Solids Struct. 41, 827 (2004).

[23] N. Saad-Gouider, R. Estevez, C. Olagnon, and R. Séguéla, Eng. Fract. Mech. 73, 2503 (2006). 